\title{
Letters to the
}

\section{Editor}

\section{Severe mitral regurgitation after percutaneous transmitral commissurotomy: Underestimated subvalvular disease To the Editor:}

We read with interest the article by Varma and associates ${ }^{1}$ in which the authors describe 23 cases of severe acute mitral regurgitation (MR) after percutaneous transmitral commissurotomy (PTMC). Tearing of either the anterior $(n=20)$ or posterior $(\mathrm{n}=3)$ mitral leaflet was responsible for production of acute MR. The authors also found that the majority of these patients had significant subvalvular disease, which was underestimated on transthoracic echocardiography.

We had a similar experience, as reported earlier. ${ }^{2}$ Acute, severe MR developed in 25 of 752 patients undergoing PTMC and necessitated emergency mitral valve replacement or repair. The most common lesion responsible for acute MR was a radial tear in either the anterior $(\mathrm{n}=$ 16) or posterior $(n=2)$ mitral leaflet. Three patients had rupture of one or more chordae. In the remaining 4 patients, creation of a pseudo-orifice in the mitral apparatus was responsible for acute MR. Moderate-to-severe commissural fusion was present in all cases, and commissural fracture was seen in only 1 case, suggesting very serious commissural fusion. The most interesting finding was the presence of moderate $(n=14)$ or severe $(n=5)$ subvalvular fusion as against pre-PTMC transthoracic echocardiographic findings of no subvalvular disease in 13 patients, mild disease in 7 patients, and moderate disease in 5 patients.

We believe the presence of undiagnosed, significant subvalvular disease may lead to either faulty transmission of balloon pressure forces leading to leaflet tear or improper engagement of the balloon leading to chordal rupture or pseudo-orifice creation. Thus, the presence of subvalvular disease plays a major role in the production of these lesions. Unfortunately, transthoracic echocardiography may not be a sen- sitive tool to evaluate subvalvular disease, as it was missed in most of our patients.

\author{
Shiv Kumar Choudhary, MCh \\ Sachin Talwar, MCh \\ Panangipalli Venugopal, MCh \\ Cardiothoracic Centre \\ All India Institute of Medical Sciences \\ New Delhi, India
}

\section{References}

1. Varma PK, Theodore S, Neema PK, Ramachandran P, Sivadasaanpillai H, Nair KK et al. Emergency surgery after percutaneous transmitral comissurotomy: operative versus echocardiographic findings, mechanisms of complications, and outcomes. J Thorac Cardiovasc Surg. 2005;130:772-6.

2. Choudhary SK, Bhan A, Sharma R, Airan B, Das B, Kumar AS, et al. Pathology of severe mitral regurgitation following balloon valvuloplasty. Asian Cardiovasc Thorac Ann. 1997; $5: 25-30$.

doi:10.1016/j.jtcvs.2005.10.044

\section{Reply to the Editor:}

We thank Choudhary and his colleagues for their interest in our article. ${ }^{1}$ Experience in our center and also reported by others ${ }^{2}$ shows that the majority of patients with severe subvalvular disease achieve a satisfactory outcome with percutaneous transmitral commissurotomy (PTMC). Hence we believe that we have to look beyond the role of undiagnosed subvalvular disease as the sole mechanism in producing mitral regurgitation (MR) after PTMC. This allows us to further clarify our hypothesis.

In 14 cases of MR that we analyzed, only 10 involved severe subvalvular disease. However, all had severe commissural fusion. In PTMC, commissural splitting is the mechanism for relieving mitral stenosis. Normally, commissures split at the point of least resistance to balloon pressure. However, in the presence of such rigid commissures, areas with lesser resistance can "give way." The posterior leaflet is often rolled up and thickened, whereas a more pliable anterior leaflet becomes vulnerable. In the series described by Choudhary 